\title{
Fusarium graminearum Chemotype-Spring Wheat Genotype Interaction Effects in Type I and II Resistance Response Assays
}

\author{
Mitra Serajazari, Kerin Hudson, Mina Kaviani, and Alireza Navabi ${ }^{\dagger}$ \\ Department of Plant Agriculture, University of Guelph, Guelph, ON Canada. \\ Accepted for publication 11 November 2018.
}

\begin{abstract}
Fusarium head blight (FHB), caused by several Fusarium spp., is a worldwide problem that severely impacts cereal grain yield and poses major risks to human and animal health due to production of the mycotoxin deoxynivalenol (DON) and its acetylated forms, 3-acetylDON (3-ADON) and 15-acetyl-DON (15-ADON). Recent studies suggest an inconsistent effect of $F$. graminearum chemotypes and resistance of wheat (Triticum aestivum) genotypes. To gain insight into the interaction effects of $F$. graminearum chemotypes and spring wheat genotypes on FHB resistance response, 10 spring wheat genotypes with varying levels of FHB resistance were inoculated with $10 \mathrm{~F}$. graminearum isolates, consisting of 53 -ADON- and 515 ADON-producing isolates and evaluated in type I (spray inoculation)

carrying the resistance allele of the Fhbl quantitative trait locus on chromosome 3BS had lower disease in type II evaluations, regardless of $F$. graminearum isolate or chemotype. Isolates of $F$. graminearum were also significantly different for disease aggressiveness. In addition, the 3 -ADON-producing isolates were $18 \%$ more aggressive than the 15-ADON isolates in type I resistance assays. No difference in aggressiveness of the two chemotypes was observed, when tested in type II resistance assays. There was no interaction effect between $F$. graminearum chemotypes and spring wheat genotypes, suggesting that screening of germplasm for resistance can be performed with limited number of aggressive isolates.
\end{abstract} and type II (point inoculation) resistance assays. Wheat genotypes
Keywords: 3-ADON, 15-ADON, Fusarium head blight, wheat.
In most wheat (Triticum aestivum L.)-producing areas of the world, Fusarium head blight (FHB, also known as head scab) is a major threat to grain yield and quality, as well as to human health. This fungal disease, caused by several species of Fusarium, mainly Fusarium graminearum Schwabe in North America, was first reported in the late 19th century in the United States (Arthur 1891; Chester 1890). Every 4 or 5 years, a severe FHB epidemic occurs in the United States, China, European Union, United Kingdom, Africa, and Brazil and the estimated yield loss in the United States between 1990s and 2008 was US\$ 3 billion (Schumann and D' Arcy 2009). In Canada, six FHB epidemics were reported from 1940 to 1980 (Sutton 1982). In addition, the FHB incidence reached $70 \%$ in an epidemic year in south-central Manitoba (Shaner 2003). Over a 20-year period, economic losses due to FHB in Canada were estimated at $\$ 50$ million to $\$ 300$ million annually (Milligan 2014).

Apart from reducing grain yield, $F$. graminearum produces trichothecene mycotoxins such as deoxynivalenol (DON) in the grain. The DON-producing strains of $F$. graminearum can be divided into 3-acetyl-DON (3-ADON)- and 15-acetyl-DON (15-ADON)producing chemotypes, based on the addition of an acetyl group to $\mathrm{C} 3$ or $\mathrm{C} 15$ in the trichothecene biosynthesis pathway. Molecular markers detecting 15-O-acetyltransferase (TRI3) and a trichothecene efflux pump (TRI12) genes have often been used to determine 3-ADON and 15-ADON chemotypes (Starkey et al. 2007). In a recent study, the toxicity of 3-ADON and 15-ADON chemotypes was evaluated using a lethal dose that kills $50 \%$ of a mice population (LD50). Oral administration of infected wheat grains containing 3-ADON or 15ADON to mice revealed significantly higher toxicity of 3-ADON

${ }^{\dagger}$ Corresponding author: A. Navabi; E-mail: anavabi@uoguelph.ca

Funding: Financial support was provided by the project by SeCan, the Grain Farmers of Ontario, and the Agriculture Adaptation Council through the Growing Forward program.

The author(s) declare no conflict of interest.

(c) 2019 The American Phytopathological Society
$(\mathrm{LD} 50=34 \mathrm{mg} / \mathrm{kg})$ compared with 15-ADON $(\mathrm{LD} 50=49 \mathrm{mg} / \mathrm{kg})$ (Asam et al. 2017). In addition, disease surveys across Canada suggest that the $F$. graminearum $15-\mathrm{ADON}$ chemotype is being replaced by the 3-ADON chemotype (Barker 2009). Analyses of trichothecene genotypes of Gibberella zeae (anamorph: $F$. graminearum) collected from winter wheat fields in the eastern parts of the United States revealed an increasing gradient in 3ADON distribution from south to north and closer to Canada (Schmale et al. 2011). A European database of F. graminearum trichothecene isolates indicted that, from 1,147 strains of F. graminearum collected from 17 European countries, 15-ADON $(82.9 \%)$ was the main chemotype, followed by $3-\mathrm{ADON}(13.6 \%)$ and nivalenol (NIV) (3.5\%) (Pasquali et al. 2016).

Breeding for resistance against FHB is very complicated due to different types of resistance against the pathogen. Studies of FHB resistance in wheat have determined five types of resistance mechanisms (He et al. 2016; Mesterházy et al. 2015). These consist of resistance to initial infection (type I), resistance to Fusarium sp. spread in spikes (type II) (Schroeder and Christensen 1963), resistance to accumulation of mycotoxins (type III), resistance to kernel infection (type IV), and resistance to yield loss (type V) (He et al. 2016; Lemmens et al. 2005; Mesterházy 1995, 2003; Miller and Arnison 1986). In practice, type I resistance can be measured following spray inoculation with $F$. graminearum based on the infected heads per plots under the field environment (Bai et al. 2018; Buerstmayr and Buerstmayr 2015) or based on the number of infected spikelets per head under controlled environment (Li et al. 2008; Schroeder and Christensen 1963; Shin et al. 2014; Yan et al. 2011; Yu et al. 2008). Yu et al. (2008) evaluated type I resistance only 7 days after spray inoculation prior to spread of the infection to neighboring florets (type II resistance).

Type II resistance is often screened for following point inoculation of spikelets based on the ratio or percentage of infection in one head to total number of spikelets over time (Bai et al. 1999). Type III and type IV resistances are examined postharvest and can be evaluated by measuring DON contents (type III) in the kernels and counting Fusarium-damaged kernels (FDKs) (type IV) (He et al. 2016). 
Genetic studies have identified several quantitative trait loci (QTL), which are effective in resistance to FHB (Buerstmayr et al. 2009) and in reduction of FDK and DON content; however, most of them appeared to be population specific (Mesterházy et al. 2015). The most nonspecific QTL with large effect are 3BS (Fhbl) and 5AS (Qfhs.ifa-5A) (Mesterházy et al. 2015). These two QTL together confer type I and II resistance to spray inoculation (Mesterházy et al. 2015). The spring wheat variety Sumai 3 from China, which carries the FHB-resistance QTL (Fhbl) on chromosome 3BS, has been a source of FHB resistance for many years and in many breeding programs (Bernardo et al. 2012). The Fhbl QTL confers resistance to Fusarium spp. spread in wheat spikes (type II resistance). Recently, a Kompetitive allele-specific (KASP) marker was developed to detect $F h b 1$ in wheat lines (Bernardo et al. 2012). The Qfhs.ifa.5A QTL, which confers resistance to Fusarium initial infection (type I resistance), was mapped to chromosome 5A using an $\mathrm{F}_{1}$-derived doubled-haploid (DH) population, resulting from a cross between CM-82036 (resistant) and Remus (susceptible) (Buerstmayr et al. 2003).

For the purpose of this study, we define the main effect of the pathogen isolates as the aggressiveness of a set of given isolates, measured quantitatively as the ability of each isolate to colonize plant tissue. We define the main effect of the host genotype as resistance, measured quantitatively as the ability of the host plant to reduce colonization by the pathogen. We also define the host genotype-pathogen isolate interaction effect as the differential response of wheat genotypes against a given set of pathogen isolates. We are specifically concerned with interaction effect, when it is associated with genotypic rank change, following inoculation with a given set of pathogen isolates.

Previous studies attempting to examine the main and interaction effects of $F$. graminearum chemotypes with wheat genotypes have revealed conflicting results. For example, a study of chemotype aggressiveness, following artificial inoculations in the field across Canada and Europe, reported no significant difference between aggressiveness of the 3-ADON and 15-ADON chemotypes (von der Ohe et al. 2010). Another study of chemotype aggressiveness and the interaction between DON derivatives and NIV-producing isolates on a range of wheat lines with different responses to FHB found stable resistance to FHB in highly resistant wheat lines carrying the 3BS QTL, regardless of $F$. graminearum chemotype (Foroud et al. 2012).

A transcriptome analysis of Sumai 3, following inoculation with 3-ADON and 15-ADON chemotypes (two florets of each wheat head with $10 \mu \mathrm{l}$ of $\left.1 \times 10^{5} \mathrm{conidia} / \mathrm{ml}\right)$, found seven putative

TABLE 1. Spring wheat genotypes used for type I and II resistance experiments

\begin{tabular}{|c|c|c|}
\hline Wheat genotype & Reaction $^{\mathrm{a}}$ & Source \\
\hline AAC Brandon & MR & Cuthbert et al. 2016 \\
\hline AAC Scotia & MR & $\begin{array}{l}\text { Ontario Spring Cereal Performance Trials } \\
2014\end{array}$ \\
\hline Carberry & MR/MS & $\begin{array}{l}\text { DePauw et al. 2011; Ontario Spring Cereal } \\
\text { Performance Trials 2014, } 2015\end{array}$ \\
\hline Frontana & $\mathrm{R}$ & Bai and Shaner 2004 \\
\hline Norwell & MS & $\begin{array}{l}\text { Ontario Spring Cereal Performance Trials } \\
2014\end{array}$ \\
\hline Peace & MS & Humphreys et al. 2014 \\
\hline Sable & HS & $\begin{array}{l}\text { Ontario Spring Cereal Performance Trials } \\
2014\end{array}$ \\
\hline SS Blomidon & S & $\begin{array}{l}\text { Ontario Spring Cereal Performance Trials } \\
2015\end{array}$ \\
\hline Sumai 3 & $\mathrm{R}$ & Bai and Shaner 2004 \\
\hline Wilkin & $S$ & $\begin{array}{l}\text { Ontario Spring Cereal Performance Trials } \\
2014\end{array}$ \\
\hline
\end{tabular}

a Field reaction to Fusarium head blight: $\mathrm{MR}=$ moderately resistant, $\mathrm{MS}=$ moderately susceptible, $\mathrm{R}=$ resistant, $\mathrm{HS}=$ highly susceptible, and $\mathrm{S}=$ susceptible. defense-related genes involved in Fusarium sp.-wheat interaction (Al-Taweel et al. 2014). This study also suggested that chemotype plays an important role in FHB resistance. Defense-related genes (e.g., UDP-glucosyltransferase, thaumatin-like protein, glucosidase, chitinase III, and peroxidase) were expressed in plants inoculated with 3-ADON and 15-ADON 48 to $144 \mathrm{~h}$ after inoculation but the quantity of the expression was higher, when inoculated with 15 -ADON. The authors hypothesized that higher expression of defense-related genes plays a role in the suppression of virulence in $15-\mathrm{ADON}$ chemotypes, which may explain some of the reports of higher aggressiveness of 3-ADON isolates.

In contrast, a chemotype aggressiveness study using 10 isolates of 3-ADON and 15-ADON chemotypes on resistant cultivar ND 2710, moderately resistant Steele-ND, and susceptible Grandin found no significant difference between the 3-ADON and 15-ADON in terms of their aggressiveness, when tested for type II resistance (Puri et al. 2016). Analysis of type I and type II resistance by inoculating susceptible cultivar Roblin and the moderately resistant $5602 \mathrm{HR}$ with $58 \mathrm{~F}$. graminearum isolates of 3-ADON or 15-ADON chemotypes, collected from Manitoba, Saskatchewan, and Alberta in Canada, found no significant difference between aggressiveness of 3-ADON and 15-ADON (Gilbert et al. 2010). Also, quantification of DON and ADON following point inoculation of Roblin and 5602-HR with $40 \mathrm{~F}$. graminearum isolates of 3-ADON or 15-ADON chemotypes, collected from Manitoba, revealed a significant interaction between wheat variety and chemotype for DON ( $P \leq$ 0.05 ) but not for ADON content (Gilbert et al. 2010).

Given the importance of an understanding of the main and interaction effects of the $F$. graminearum chemotypes and wheat genotypes and to resolve the discrepancies indicated above, we hypothesized that different spring wheat genotypes, with varying levels of resistance against FHB, would respond differently, when inoculated with a diverse set of 3-ADON- and 15-ADON-producing isolates of $F$. graminearum from across Canada. Therefore, the objectives of this research were to (i) examine the aggressiveness of 3-ADON- and 15-ADON-producing isolates, (ii) examine the resistance response of wheat genotypes with and without the Fhbl QTL, and (iii) study the differential responses of wheat genotypes against 3-ADON- and 15-ADON-producing isolates of $F$. graminearum following point and spray inoculation. Such interactions can have implications in designing selection strategies for breeding FHB-resistant varieties.

\section{MATERIALS AND METHODS}

Plant materials and growth conditions. Based on prior field FHB responses, 10 spring wheat genotypes were selected for experiments (Table 1). Seeds were planted in a mixture (1:1) of Sunshine LA4 (Sungrow Horticulture; Seba Beach, AB, Canada) and coarse Turface (Turface Athletics; Buffalo Grove, IL, U.S.A.) in 1.5 by 8.25 in. cones (SC10; Greenhouse Megastor, Danville, IL, U.S.A.). Plants were raised in a controlled-environment growth room at the University of Guelph, ON, Canada, with a photoperiod of $16 \mathrm{~h}$ of light and $8 \mathrm{~h}$ of darkness, a temperature of 20 and $18^{\circ} \mathrm{C}$, and a relative humidity of $60 \%$ prior to inoculation. After inoculation, growth conditions were a photoperiod of $16 \mathrm{~h}$ of light and $8 \mathrm{~h}$ of darkness, a temperature of 26 and $24^{\circ} \mathrm{C}$, and a relative humidity of $80 \%$.

Preparation of $\boldsymbol{F}$. graminearum inoculum. A collection of $10 \mathrm{~F}$. graminearum isolates was obtained from the Canadian Grain Commission (Winnipeg, MB, Canada). This collection, which consists of five 3-ADON and five 15-ADON chemotypes (Table 2), has been isolated from Ontario, Alberta, Saskatchewan, and Manitoba from 2006 to 2010. The isolates were cultured on potato dextrose agar for 7 days and a 5-mm agar disc was transferred to a 250-ml flask containing a sterilized mixture of wheat straw $(5 \mathrm{~g})$ and $\mathrm{dH}_{2} \mathrm{O}(125 \mathrm{ml})$. The inoculated flasks were placed on a shaker $(120 \mathrm{rpm})$ at $25^{\circ} \mathrm{C}$ in the dark for 10 days. Macroconidia were 
harvested by passing the culture through three layers of cheesecloth. Macroconidia concentration was adjusted to 50,000 macroconidia/ $\mathrm{ml}$. Each plant head was inoculated with a single chemotype.

Chemotype and genotype classifications using molecular markers. Genomic DNA of $F$. graminearum isolates were extracted from $50 \mathrm{mg}$ of mycelia using DNeasy Plant Mini Spin Columns (Qiagen, Germantown, MD, U.S.A.). Chemotypespecific primers were used in a multiplex polymerase chain reaction (PCR) to identify 3-ADON and 15-ADON chemotypes as previously described by Starkey et al. (2007). The PCR products were size fractionated and visualized using the QIAxcel Advanced System (QIAGEN GmbH, Hilden, Germany).

A single-nucleotide polymorphism marker for $F h b 1$, snp3BS-8, was used to identify whether the $F h b 1$ resistance allele was present and was analyzed to verify the Fhbl-resistant allele (Bernardo et al. 2012). The KASP assay was used to detect and distinguish resistant and susceptible alleles for $F h b 1$. Polymorphism was identified using the LGC Genomics (https://www.lgcgroup.com) KASP system fluorescent assay with two allele-specific primers, susceptible primer (5'-CACATGCATTTGCAAGGTTGTTATCC-3') and resistant primer (5'-CACATGCATTTGCAAGGTTGTTATCG$3^{\prime}$ ), and one common (reverse) primer (5'-CAAAGCAGCCTTA GGTCAATAGTTTGAAA-3') (Bernardo et al. 2012). PCR was run according to the LGC instructions using a reaction volume of $10 \mu \mathrm{l}$, which consisted of $5 \mu \mathrm{l}$ of $2 \times$ KASP reaction mix, $0.14 \mu \mathrm{l}$ of $72 \times$ assay mix, and $5 \mu \mathrm{l}$ of template DNA $\left(10 \mathrm{ng} \mu \mathrm{l}^{-1}\right)$. Endpoint genotyping was conducted in QuantStudio 6 Flex System (Applied Biosystems, Foster City, CA, U.S.A.).

Evaluation of wheat genotypes for type II resistance. Two experiments were conducted to evaluate wheat genotypes for type II resistance. A preliminary experiment (experiment 1) was performed to determine the efficiency of point inoculation in our experimental system (see "Plant materials and growth conditions"). In this preliminary experiment, Sumai 3, Frontana, Agriculture Adaptation Council (AAC) Brandon, AAC Scotia, Carberry, Peace, Sable, and Wilkin were planted in four replications. Later, in the second experiment, the same spring wheat genotypes as well as SS Blomidon and Norwell were planted in eight replications. At anthesis, each genotype was separately point inoculated with each one of the 10 $F$. graminearum isolates, of which 5 isolates were 3-ADON- and 5 were 15 -ADON-producing chemotypes. For point inoculation, the right floret of the middle spikelet was injected with $10 \mu \mathrm{l}$ of a macroconidia suspension (50,000 macroconidia/ml) using a micropipette. After inoculation, the spikes were immediately covered with plastic bags and the plants were transferred to a growth room with a photoperiod of $16 \mathrm{~h}$ of light and $8 \mathrm{~h}$ of darkness, a temperature of 26 and $24^{\circ} \mathrm{C}$, and a relative humidity of $80 \%$. The number of infected spikelets in each head was recorded 7,14 , and 21 days after inoculation (dai) in the first experiment and 5,10 , and 15 dai in the second experiment. The area under the disease progress curve (AUDPC) was calculated according to the following equation, where $Y_{i}$ is disease level at time $t_{i}$.

$$
A U D P C=\sum_{i=1}^{a}\left[\left\{\frac{\left(Y_{i}+Y_{(i+1)}\right)}{2}\right\}\left(t_{(i+1)-} t_{i}\right)\right]
$$

Evaluation of wheat genotypes for type I resistance. To determine type I resistance, experimental plants, $F$. graminearum isolates, and growth conditions were similar to the second experiment for type II resistance, except that each plant head was sprayed with $2 \mathrm{ml}$ of a suspension of macroconidia $(50,000$ macroconidia $/ \mathrm{ml}$ ) at anthesis. The inoculated heads were covered with plastic bags for 5 days. Plants were kept in a growth room with a photoperiod of $16 \mathrm{~h}$ of light and $8 \mathrm{~h}$ of darkness, a temperature of 26 and $24^{\circ} \mathrm{C}$, and a relative humidity of $80 \%$. Resistance to initial infection (type I resistance) under a controlled environment was measured according to Yan et al. (2011); Yu et al. (2008), and Schroeder and Christensen (1963) by counting the number of infected spikelets in each head only one time 5 days after spray inoculation prior to spread of the pathogen to neighboring spikelets.

Statistical analysis. All experiments were performed using a randomized complete block design. Data were analyzed in the PROC MIXED procedure in SAS (V 9.4; SAS Institute Inc., Cary, NC, U.S.A.) for each test separately, in which the main effects of isolate and genotype and the genotype-isolate interaction were considered fixed effects and block was considered random effect. The main effect of pathogen isolate and wheat genotype examines the aggressiveness of the pathogen and resistance response of the host, while the interaction effect examines the isolate-specific response of the genotypes. Random and independent distribution of residuals were tested by plotting the studentized residuals against predicted values, while normality of the residuals were tested using the Shapiro-Wilks test in the PROC UNIVARIATE procedure in SAS. Data for type I and type II responses were transformed using square root and logarithmic transformations, respectively. Leastsquared means were estimated using the LSMEANS statement in PROC MIXED. Pairwise comparisons were conducted using the PDIFF statement. The least-squared means were then backtransformed. Single-degree of freedom contrasts were performed to test the hypotheses that the 3-ADON and 15-ADON isolates are similar for aggressiveness and that the genotypes with and without $F h b l$ are similar for disease severity for type II or disease damage for type I resistance. For each test, a genotype-isolate matrix was constructed using the back-transformed least-squared mean values. Each matrix was then subjected to a principal component (PC) analysis in PRINCOMP followed by PRINQUAL procedures in SAS. The biplot of the PC1 and PC2 eigenvalues was constructed and the proportion of variance accounted for by each PC was estimated for each test. Different chemotypes and genotypes with and without $F h b 1$ were color coded in the biplots.

\section{RESULTS}

Classification of genotypes and chemotypes using molecular markers. Ten $F$. graminearum isolates used in the experiments were grouped into 3-ADON- and 15-ADONproducing chemotypes using TRI3 and TRI12 markers (Table 2). The KASP assay identified the presence of an Fhbl resistant allele in Sumai 3, Carberry, and AAC Brandon.

Evaluation of type II and type I resistance. In experiments 1 and 2 (type II resistance), the main effect of wheat genotypes (resistance) as well as the difference between genotypes with and without the $F h b 1$ marker were significant $(P<0.0001)$. The main effect of $F$. graminearum isolates (aggressiveness) was only significant in experiment 2 . However, the differences between the 3-ADON and 15-ADON chemotypes and the isolate-wheat genotype interaction effects were not significant (Table 3). Evaluation of type I response, on the other hand, revealed a significant difference $(P<0.0001)$ in the wheat genotype main effect, $F$. graminearum

TABLE 2. The collection of 3-acetyl-deoxynivalenol (ADON)- and 15-ADONproducing Fusarium graminearum isolates used for type I and II assays

\begin{tabular}{llcll}
\hline Isolate ID & \multicolumn{1}{c}{ Province } & Year & Isolate & Chemotype \\
\hline ON-06-146 & Ontario & 2006 & F. graminearum & 3-ADON \\
ON-06-211 & Ontario & 2006 & F. graminearum & 3-ADON \\
A7-10-011 & Alberta & 2010 & F. graminearum & 3-ADON \\
S4B-10-007 & Saskatchewan & 2010 & F. graminearum & 3-ADON \\
Grass 10-3 & Manitoba & 2010 & F. graminearum & 3-ADON \\
ON-06-007 & Ontario & 2006 & F. graminearum & 15-ADON \\
ON-06-052 & Ontario & 2006 & F. graminearum & 15-ADON \\
A6-10-007 & Alberta & 2010 & F. graminearum & 15-ADON \\
S4A-10-002 & Saskatchewan & 2010 & F. graminearum & 15-ADON \\
Grass 13-4 & Manitoba & 2010 & F. graminearum & 15-ADON \\
\hline
\end{tabular}


isolates, as well as the aggressiveness of the 3-ADON and 15ADON chemotypes. The isolates of the 3-ADON chemotype were found to be more aggressive than $15-\mathrm{ADON}$, causing $18 \%$ higher final disease damage (Table 4). This difference between the aggressiveness of the 3-ADON and 15-ADON chemotypes did not hold, when the wheat genotypes were evaluated for type II resistance (Tables 3 and 4).

In experiment 1 , wheat genotypes containing the Fhbl marker showed 60 and $50 \%$ fewer disease symptoms, when inoculated with
3-ADON- and 15-ADON-producing isolates, respectively. In experiment 2 , these reductions in disease symptoms were 50 and $30 \%$, respectively (Table 4). As expected, the effect of the Fhb1 marker on type I response evaluation was not significant regardless of isolate chemotype. Also, in all three experiments, Wilkin was the most susceptible and Sumai 3 was the most resistant line (Fig. 1).

In PC analyses (Fig. 2), separately conducted for the three experiments, PC1 accounted for 54 to $64 \%$ of the variation and PC2 accounted for 13 to $17 \%$ of the variation, resulting in the biplots of

TABLE 3. Mixed-model analysis of variance of type I and type II response of 10 wheat genotypes against 10 isolates of 3-acetyl-deoxynivalenol (ADON)- and 15-ADON-producing Fusarium graminearum

\begin{tabular}{|c|c|c|c|c|c|c|}
\hline \multirow[b]{3}{*}{ Fixed effects } & & & \multicolumn{4}{|c|}{ Type II } \\
\hline & \multicolumn{2}{|c|}{ Type I } & \multicolumn{2}{|c|}{ Experiment 1} & \multicolumn{2}{|c|}{ Experiment 2} \\
\hline & $F$ value & $P$ value & $F$ value & $P$ value & $F$ value & $P$ value \\
\hline $3 \mathrm{~A}-$ versus $15-\mathrm{ADON}$ & 20.50 & $<0.0001$ & 0.48 & 0.4905 & 0.01 & 0.9064 \\
\hline Genotype & 8.51 & $<0.0001$ & 13.35 & $<0.0001$ & 11.05 & $<0.0001$ \\
\hline Fhbl+ versus Fhbl- & 3.22 & 0.0734 & 69.54 & $<0.0001$ & 55.99 & $<0.0001$ \\
\hline Isolate $\times$ genotype & 1.06 & 0.3569 & 0.81 & 0.828 & 1.1 & 0.2691 \\
\hline
\end{tabular}

TABLE 4. Least-squared means of the disease severity of 10 spring wheat genotypes for type II and disease damage for type I resistance following inoculation with 10 isolates of 3-acetyl-deoxynivalenol (ADON)- and 15-ADON-producing Fusarium graminearum

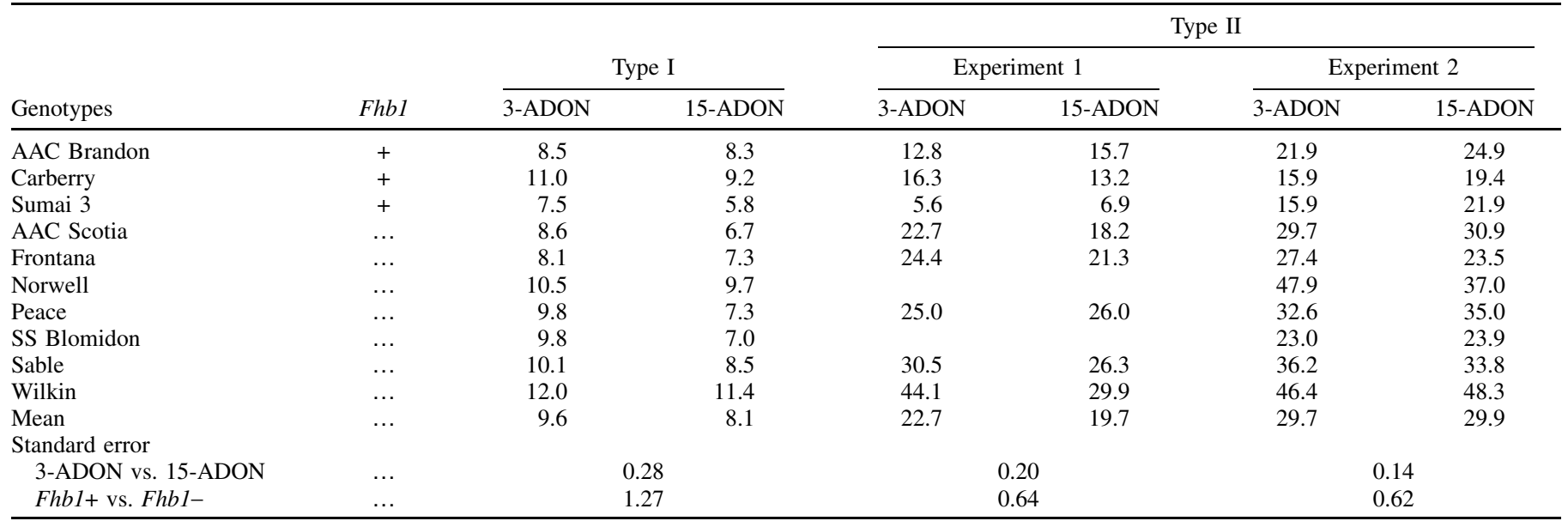
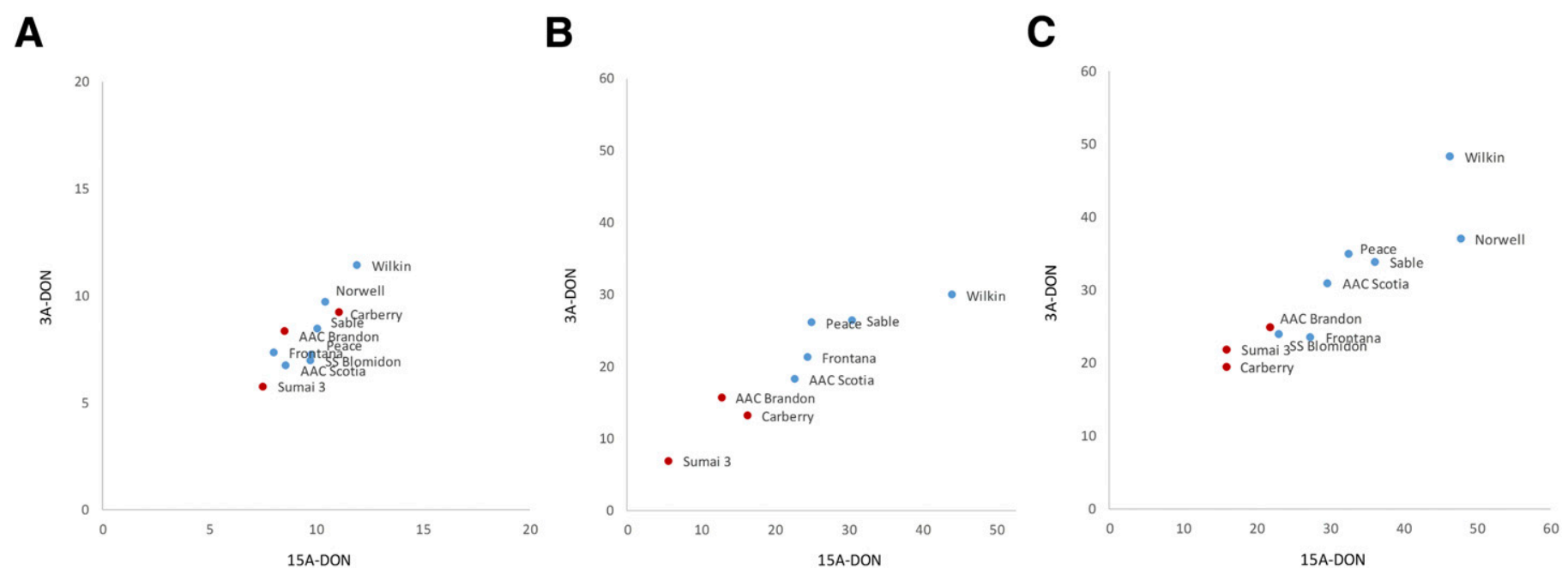

Fig. 1. Disease severity for type II and disease damage for type I resistance of spring wheat genotypes inoculated with 3-acetyl-deoxynivalenol (ADON) (Y axis) and 15-ADON (X axis) chemotypes. A, Type I resistance, B, type II resistance in experiment 1, and $\mathbf{C}$, type II resistance in experiment 2. Dark gray/red dots represent genotypes carrying the resistant allele of the Fhb1 QTL. Light gray/blue dots represent genotypes lacking the resistant allele of the Fhb1 QTL. 
PC1 and PC2 accounting for 73 to $78 \%$ of total variation. In both type II experiments (experiment 1 and experiment 2), PC1 separated the main effect of wheat genotypes, with those carrying the Fhbl marker falling on the left side of the origin and genotypes without the Fhbl marker on the right side of the origin. In both experiments, except for one isolate, PC2 separated the main effect of the $F$. graminearum isolate. The only meaningful clustering that was found in the biplots of experiments 1 and 2 was clustering of the genotypes carrying the $F h b 1$ marker, known to contribute to type II resistance. No clustering was found in these biplots to indicate that wheat genotypes interact differently with the two chemotypes. In the biplot of the type I evaluation, PC1 mainly separated the effect of genotypes and PC2 separated the effect of isolates. However, no clustering of genotypes, based on their Fhbl genotype, or clustering of isolates, based on their DON chemotypes, was observed. Results of the three biplots did not point to any meaningful interaction between DON chemotype and wheat genotype.

In all three biplots, $F$. graminearum isolates had positive relationships except for one isolate in type I evaluation and one isolate in experiment 2 of type II evaluation (Fig. 2A, B, and D). In experiment 2, the 15-ADON-producing isolate ON-06-007 had a negative relationship with S4A-10-002, ON-06-52, and A7-10-011. All other isolates had positive relationships (Fig. 2C). For type I resistance, ON-06-211 had a negative relationship with Grass-13-4, Grass-10-3, and ON-06-52.

\section{DISCUSSION}

We examined a set of 3-ADON- and 15-ADON-producing isolates of $F$. graminearum on a set of wheat genotypes with different levels of resistance response in order to study variation in aggressiveness of the pathogen as it relates to chemotype, as well as isolate- or chemotype-specific reaction of the host genotypes.

The main effect of wheat genotype was always significant. In type II evaluations, the major contributor to this difference seems to be the presence or absence of Fhbl QTL, regardless of $F$. graminearum chemotype. Molecular marker analysis showed that Fhbl QTL is involved in type II resistance, which is in agreement with previous reports (Bai et al. 1999; Zhou et al. 2002). In our experiments, the resistant lines carrying the $F h b 1$ marker showed resistance to both 3-ADON- and 15-ADON-producing chemotypes. It was shown that plants carrying $F h b 1$ QTL are able to detoxify DON by producing DON-glycoside (Lemmens et al. 2005), resulting in higher resistance in plants with lower DON (Mesterházy et al. 2015). Recently, mutation analysis, gene silencing, and transgenic overexpression showed that a poreforming toxin-like (PFT) gene located in Fhbl QTL causes resistance to FHB (Rawat et al. 2016). Foroud et al. (2012) evaluated two DH populations resulting from crosses between Superb (no resistance QTL) and CM82036 (3BS and 5A QTL) against type II resistance with point inoculation. Their results showed that DH populations were resistant to all chemotypes (3ADON, 15-ADON, and NIV). They also showed that susceptible lines with no resistance QTL (Superb, Crystal, and CIMMYT 11) accumulated higher trichothecene compared with resistant lines and, interestingly, they accumulated 3-ADON more than 15-ADON and NIV (Foroud et al. 2012). We conducted type I evaluations only once, 5 days after spray inoculation. Evaluation of type I resistance in this manner prevented confounding type I and type II resistance (Schroeder and Christensen 1963; Yan et al. 2011; Yu et al. 2008). This evaluation is different than type I evaluation under field conditions, where it is measured based on the number of infected heads per plot, as described by Bai et al. (2018). In our type I evaluation, the presence and absence of the $F h b 1$ marker was not the major determinant and wheat genotypes had a range of reactions to F. graminearum isolates regardless of their chemotype. Moreover, $3-\mathrm{ADON}$ isolates were more aggressive compared with 15-ADON. von der Ohe et al. (2010) and Gilbert et al. (2010) found no significant difference between the aggressiveness of 3-ADON and 15-ADON related to the field resistance.

In all experiments, Wilkin was the most susceptible and Sumai 3 was the most resistant genotype (Fig. 1). Sable was previously reported as highly susceptible under field inoculation (Table 1) but it was less susceptible than Wilkin in our experiments. This difference in susceptibly of Wilkin may be due to the experimental conditions. In this regard, artificial inoculations under Ontario field conditions were conducted using a mixture of $F$. graminearum isolates (Table 1), whereas our experiments were conducted using a single chemotype for artificial inoculation under controlledenvironment growth rooms. Also, FHB field experiments are subjected to a genotype-environment effect. This interaction effect under field conditions cannot be compared with that under our controlled-environment condition. Our study indicates a lack of an overall significant interaction or specificity between wheat genotypes and isolates or DON chemotypes. In type II evaluations, for example, genotypes that carry some level of type II resistance (mainly due to the presence of Fhbl QTL) are resistant regardless of the isolate or chemotype they are being tested with, which is in agreement with the study conducted by Foroud et al. (2012). The same results were found for type I response, except for one isolate
A

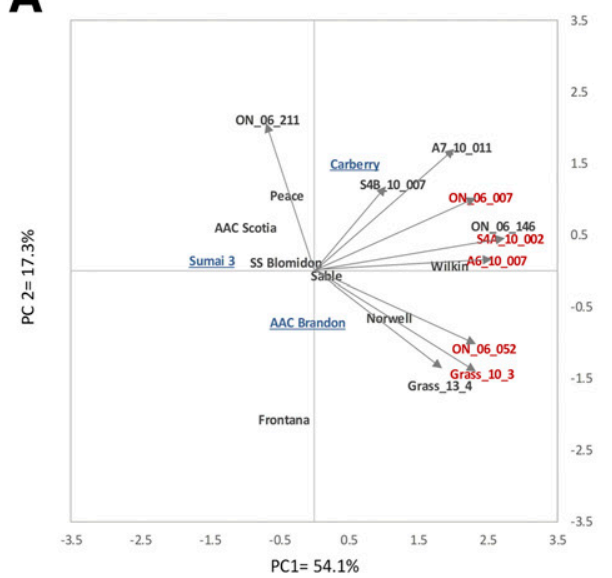

B

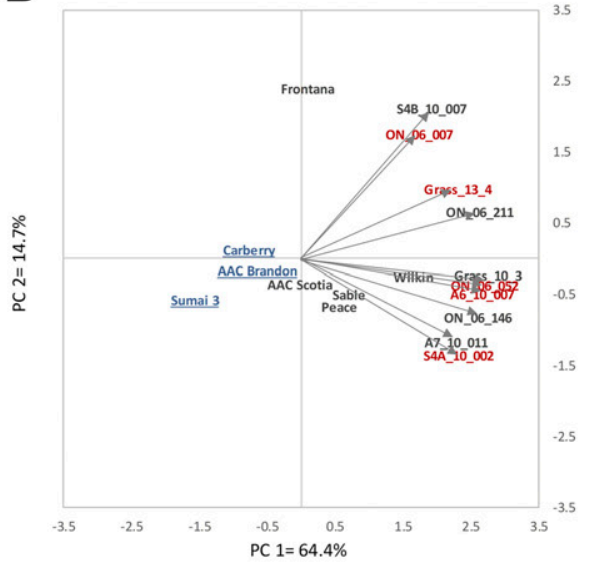

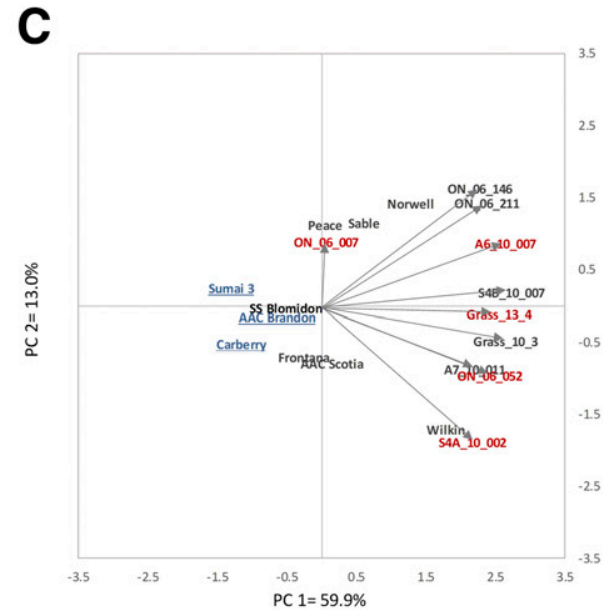

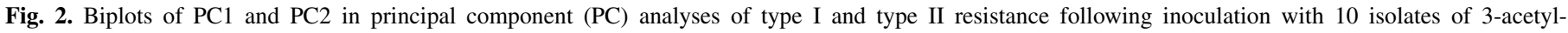



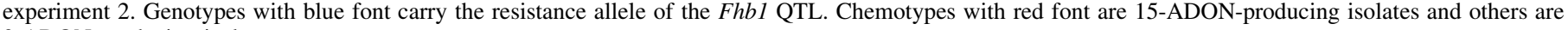
3-ADON-producing isolates. 
(ON-06-211) (Fig. 2A). The susceptible genotypes, on the other hand, and especially for type II resistance, showed some level of rank change, when tested with different isolates even though the overall interaction was not significant. Regardless of these findings, no conclusion can be made that genotypes interact differentially with the two chemotypes. The significance of this finding in the breeding programs is that, with a lack of genotype-chemotype interaction, evaluation of germplasm for resistance against $F$. graminearum can be done with a number of aggressive local isolates, regardless of their chemotype, and that any resistance found can be expected to have resistance against a broad range of $F$. graminearum isolates.

Previous studies in several cereal crops have reported the complex effects of trichothecenes on $F$. graminearum virulence as being more host specific but less chemotype specific (Maier et al. 2006). The effect of trichothecenes on $F$. graminearum virulence was investigated by disrupting the trichodiene synthase (Tri5) gene, the first gene in the trichothecene pathway, using the disruption vector $p$ Tri5_ko. The isolates with $\Delta$ Tri5 were used to inoculate wheat, barley, and maize. In wheat, the $\Delta$ Tri5 isolates produced symptoms but there was no spread of symptoms in wheat heads compared with the wild-type isolate. In barley, there was no difference between the $\Delta$ Tri5 and wild-type isolates for producing symptoms. In maize, isolates with $\Delta T r i 5$ produced the same disease symptoms compared with the wild-type isolates but isolates with disruption in the NIV-producing enzymes caused less disease compared with the wild type (Maier et al. 2006). Their results showed that Munk, a wheat variety susceptible to FHB, is different from barley and maize in response to $\Delta$ Tri5 isolates and that trichothecenes play an important role in the spread of infection in the wheat head. To investigate the differences among wheat cultivars against FHB chemotypes, we tested a range of wheat cultivars with different ranges of resistance response. Our results showed a significant difference between wheat genotypes (host) in disease damage against 3-ADON and 15-ADON but not any interaction between wheat genotypes and chemotypes.

A recent study hypothesized that $3-\mathrm{ADON}$ isolates are more aggressive than 15-ADON isolates due to faster growth and reproduction (Vujanovic et al. 2012). This hypothesis was based on the finding that 3-ADON-producing isolates from several regions of Canada grew faster than 15-ADON isolates, when exposed to extremely high or low temperatures. Therefore, the 3ADON-producing isolates can withstand harsher environmental fluctuations than 15-ADON-producing isolates. Our results show that, under a controlled environment with no environmental fluctuations, the 3-ADON-producing isolates are still more aggressive than 15-ADON-producing isolates for type I resistance (resistance against initial infection) (Table 4) but not for type II resistance (resistance against the spread of the infection within spike). This suggests that the aggressiveness of the 3-ADON chemotype is not related to environmental effects and may rather depend on higher toxicity of this chemotype. Apart from withstanding harsher environmental fluctuations, other characteristics of 3-ADON-producing isolates may be responsible for spreading in larger areas of Canada and replacing 15-ADONproducing isolates (Ward et al. 2002).

In contrast to the results reported by Vujanovic et al. (2012), a recent study of the 3-ADON-, 15-ADON-, and NIV-producing isolates of $F$. graminearum from China did not find a significant difference in growth rates of these isolates at $15^{\circ} \mathrm{C}$, although the 15 ADON-producing isolates released more ascospores (Liu et al. 2017). At 20 and $25^{\circ} \mathrm{C}$, however, 15 -ADON-producing isolates grew faster than 3-ADON- and NIV-producing isolates. In all temperatures examined, the 15-ADON-producing isolates generated more perithecia 2 to 3 days earlier than the two other chemotypes. This study also found no difference in aggressiveness between 3-ADON and 15-ADON chemotypes, when type II resistance was evaluated on a moderately resistant genotype
(Yangmai 158) but more DON was found in seed infected with the 3-ADON-producing isolates. This study suggested that the spread of $F$. graminearum chemotypes depends on their biological characteristics. In agreement with these results, our study of 10 wheat genotypes also found no significant difference between 3ADON- and 15-ADON-producing isolates for type II resistance (Table 3). Moreover, our evaluation of type I resistance revealed that 3-ADON-producing isolates were more aggressive than $15-\mathrm{ADON}$ producing isolates. We suggest that testing for type I resistance in the controlled environment will provide a better indication of resistance to $F$. graminearum chemotypes under natural wheat field conditions. If the aggressiveness of 3-ADON-producing isolates was related to their higher toxicity, then we would have expected higher aggressiveness of this chemotype for type II resistance. Indeed, we did not find a significant difference between chemotypes for type II resistance. Our results further suggest that 3-ADON production is an important factor for causing initial infection but this may be related to higher expression of defense-related genes against 15-ADON-producing chemotypes (Al-Taweel et al. 2014) rather than to higher toxicity. Mechanisms that are involved in the type I resistance are unidentified and not well defined (Mesterházy et al. 2015). Although several studies found negative correlation between anther extrusion and plant height with type I susceptibility to FHB (He et al. 2016; Lu et al. 2013; Mao et al. 2010; Skinnes et al. 2010), this type of resistance can be considered more than a morphological barrier against Fusarium sp. penetration.

In conclusion, the only significant difference between the 3ADON- and 15-ADON-producing isolates was higher aggressiveness in type I resistance, and there was no interaction between genotypes and chemotypes in both types of resistance. Type I resistance might not be considered just a physical barrier against Fusarium head blight. The more extensive spread of the 3-ADON chemotypes may be related to higher expression of defense genes against 15-ADON (Al-Taweel et al. 2014) apart from its toxicity or tolerance to environmental fluctuations. Further experiments are required to elucidate different reactions of wheat genotypes to 3ADON and 15-ADON chemotypes at the molecular levels.

\section{ACKNOWLEDGMENTS}

We thank T. Graefenhan of the Canadian Grain Commission, Winnipeg, MB, Canada, for providing the $F$. graminearum isolates; and N. Wilker, A. Harris, and K. Bosnic for their technical assistance.

\section{LITERATURE CITED}

Al-Taweel, K., Fernando, W. G. D., and Brûlé-Babel, A. L. 2014. Transcriptome profiling of wheat differentially expressed genes exposed to different chemotypes of Fusarium graminearum. Theor. Appl. Genet. 127: 1703-1718.

Arthur, J. C. 1891. Wheat scab. Indiana Agric. Exp. Stn. Bull. 36:129-138.

Asam, S., Halber, K., and Rychlik, M. 2017. Fusarium mycotoxins in food. Pages 295-336 in: Chemical Contamination and Residues in Food. D. Schrenk and A. Catrus, eds. Elsevier, Cambridge, MA.

Bai, G., Kolb, F., Shaner, G., and Domier, L. 1999. Amplified fragment length polymorphism markers linked to a major quantitative trait locus controlling scab resistance in wheat. Phytopathology 89:343-348.

Bai, G., and Shaner, G. 2004. Management and resistance in wheat and barley To Fusarium head blight. Annu. Rev. Phytopathol. 42:135-161.

Bai, G., Su, Z., and Cai, J. 2018. Wheat resistance to Fusarium head blight. Can. J. Plant Pathol. 40:336-346.

Barker, B. 2009. Fusarium head blight continues to be a challenge. Online publication. Top Crop Manager. https://www.topcropmanager.com/cereals/ fusarium-head-blight-continues-to-be-a-challenge-4434

Bernardo, A. N., Ma, H., Zhang, D., and Bai, G. 2012. Single nucleotide polymorphism in wheat chromosome region harboring $F h b 1$ for Fusarium head blight resistance. Mol. Breed. 29:477-488.

Buerstmayr, H., Ban, T., and Anderson, J. A. 2009. QTL mapping and marker assisted selection for Fusarium head blight resistance in wheat: A review. Plant Breed. 128:1-26.

Buerstmayr, H., Steiner, B., Hartl, L., Griesser, M., Angerer, N., and Lengauer, D. 2003. Molecular mapping of QTLs for Fusarium head blight resistance 
in spring wheat. II. Resistance to fungal penetration and spread. Theor. Appl. Genet. 107:503-508.

Buerstmayr, M., and Buerstmayr, H. 2015. Comparative mapping of quantitative trait loci for Fusarium head blight resistance and anther retention in the winter wheat population Capo $\times$ Arina. Theor. Appl. Genet. 128: 1519-1530.

Chester, F. D. 1890. The Scab of the Wheat. Del. Agric. Exp. Stn. 3.

Cuthbert, R. D., DePauw, R. M., Knox, R. E., Singh, A. K., McCaig, T. N., Fetch, T. 2016. AAC Elie hard red spring wheat. Can. J. Plant Sci. 97: 393-401.

DePauw, R. M., Knox, R. E., McCaig, T. N., Clarke, F. R., and Clarke, J. M. 2011. Carberry hard red spring wheat. Can. J. Plant Sci. 91:529-534.

Foroud, N. A., McCormick, S. P., MacMillan, T., Badea, A., Kendra, D. F., and Ellis, B. E. 2012. Greenhouse studies reveal increased aggressiveness of emergent Canadian Fusarium graminearum chemotypes in wheat. Plant Dis. 96:1271-1279.

Gilbert, J., Clear, R. M., Ward, T. J., Gaba, D., Tekauz, A., and Turkington, T. K. 2010. Relative aggressiveness and production of 3- or 15-acetyl deoxynivalenol and deoxynivalenol by Fusarium graminearum in spring wheat. Can. J. Plant Pathol. 32:146-152.

He, X., Lillemo, M., Shi, J., Wu, J., Bjørnstad, Å., Belova, T. 2016. QTL characterization of Fusarium head blight resistance in CIMMYT bread wheat line Soru\#1. PLoS One 11:e0158052.

Humphreys, D. G., Townley-Smith, T. F., Lukow, O. M., McCallum, B. D., Fetch, T. G., and Gilbert, J. A. 2014. Peace hard red spring wheat. Can. J. Plant Sci. 94:1297-1302.

Lemmens, M., Scholz, U., Berthiller, F., Dall'Asta, C., Koutnik, A., Schuhmacher, R., Adam, G., Buerstmayr, H., Mesterházy, A., Krska, R., and Ruckenbauer, P. 2005. The ability to detoxify the mycotoxin deoxynivalenol colocalizes with a major quantitative trait locus for Fusarium head blight resistance in wheat. Mol. Plant-Microbe Interact. 18:1318-1324.

Li, H. P., Zhang, J. B., Shi, R. P., Huang, T., Fischer, R., and Liao, Y. C. 2008. Engineering Fusarium head blight resistance in wheat by expression of a fusion protein containing a Fusarium-specific antibody and an antifungal peptide. Mol. Plant-Microbe Interact. 21:1242-1248.

Liu, Y. Y., Sun, H. Y., Li, W., Xia, Y. L., Deng, Y. Y., Zhang, A. X. 2017. Fitness of three chemotypes of Fusarium graminearum species complex in major winter wheat-producing areas of China. PLoS One 12:e0174040.

Lu, Q., Lillemo, M., Skinnes, H., He, X., Shi, J., and Ji, F. 2013. Anther extrusion and plant height are associated with type I resistance to Fusarium head blight in bread wheat line "Shanghai-3/Catbird.". Theor. Appl. Genet. 126:317-334.

Maier, F. J., Miedaner, T., Hadeler, B., Felk, A., Salomon, S., and Lemmens, M. 2006. Involvement of trichothecenes in fusarioses of wheat, barley and maize evaluated by gene disruption of the trichodiene synthase (Tri5) gene in three field isolates of different chemotype and virulence. Mol. Plant Pathol. 7:449-461.

Mao, S. L., Wei, Y. M., Cao, W., Lan, X. J., Yu, M., and Chen, Z. M. 2010. Confirmation of the relationship between plant height and Fusarium head blight resistance in wheat (Triticum aestivum L.) by QTL meta-analysis. Euphytica 174:343-356.

Mesterházy, Á. 1995. Types and components of resistance to Fusarium head blight of wheat. Plant Breed. 114:377-386.

Mesterházy, Á. 2003. Breeding wheat for Fusarium head blight resistance. Pages 211-240 in: Fusarium Head Blight of Wheat and Barley. K. J. Leonard and W. R. Bushnell, eds. American Phytopathological Society, St. Paul, MN.

Mesterházy, Á., Lehoczki-Krsjak, S., Varga, M., Szabó-Hevér, Á., Tóth, B., and Lemmens, M. 2015. Breeding for FHB resistance via Fusarium damaged kernels and deoxynivalenol accumulation as well as inoculation methods in winter wheat. Agric. Sci. 6:970-1002.

Miller, J. D., and Arnison, P. G. 1986. Degradation of deoxynivalenol by suspension cultures of the Fusarium head blight resistant wheat cultivar Frontana. Can. J. Plant Pathol. 8:147-150.
Milligan, P. 2014. Fusarium in the bin. Online publication. https://www.grainews. ca/2014/12/03/fusarium-in-the-bin/

Ontario Spring Cereal Performance Trials. 2014. Online publication. http:// www.gocereals.ca/archive/OCCC_2014_sc_performance.pdf

Ontario Spring Cereal Performance Trials. 2015. Online publication. http:// www.gocereals.ca/archive/OCCC_2015_sc_performance.pdf

Pasquali, M., Beyer, M., Logrieco, A., Audenaert, K., Balmas, V., and Basler, R. 2016. A European database of Fusarium graminearum and F. culmorum trichothecene genotypes. Front. Microbiol. 7:406.

Puri, K. D., Yan, C., Leng, Y., and Zhong, S. 2016. RNA-seq revealed differences in transcriptomes between $3 \mathrm{ADON}$ and 15ADON populations of Fusarium graminearum in vitro and in planta. PLoS One 11: e0163803.

Rawat, N., Pumphrey, M. O., Liu, S., Zhang, X., Tiwari, V. K., and Ando, K. 2016. Wheat $F h b 1$ encodes a chimeric lectin with agglutinin domains and a pore-forming toxin-like domain conferring resistance to Fusarium head blight. Nat. Genet. 48:1576-1580.

Schmale, D. G., Wood-Jones, A. K., Cowger, C., Bergstrom, G. C., and Arellano, C. 2011. Trichothecene genotypes of Gibberella zeae from winter wheat fields in the eastern USA. Plant Pathol. 60:909-917.

Schroeder, H. W., and Christensen, J. J. 1963. Factors affecting resistance of wheat to scab caused by Gibberella zeae. Phytopathology 53:831-838.

Schumann, G. L., and D'Arcy, C. J. 2009. Essential Plant Pathology. American Phytopathological Society, St. Paul, MN.

Shaner, G. 2003. Epidemiology of Fusarium head blight of small grain in North America. Pages 84-120 in: Fusarium Head Blight of Wheat and Barley. K. J. Leonard and W. R. Bushnell, eds. American Phytopathological Society, St. Paul, MN.

Shin, S., Kim, K. H., Kang, C. S., Cho, K. M., Park, C. S., and Okagaki, R. 2014. A simple method for the assessment of Fusarium head blight resistance in Korean wheat seedlings inoculated with Fusarium graminearum. Plant Pathol. J. 30:25-32.

Skinnes, H., Semagn, K., Tarkegne, Y., Marøy, A. G., and Bjørnstad, Å. 2010. The inheritance of anther extrusion in hexaploid wheat and its relationship to Fusarium head blight resistance and deoxynivalenol content. Plant Breed. 129:149-155.

Starkey, D. E., Ward, T. J., Aoki, T., Gale, L. R., Kistler, H. C., and Geiser, D. M. 2007. Global molecular surveillance reveals novel Fusarium head blight species and trichothecene toxin diversity. Fungal Genet. Biol. 44: 1191-1204.

Sutton, J. C. 1982. Epidemiology of wheat head blight and maize ear rot caused by Fusarium graminearum. Can. J. Plant Pathol. 4:195-209.

von der Ohe, C., Gauthier, V., Tamburic-Ilincic, L., Brule-Babel, A., Fernando, W. G. D., and Clear, R. 2010. A comparison of aggressiveness and deoxynivalenol production between Canadian Fusarium graminearum isolates with 3-acetyl and 15-acetyldeoxynivalenol chemotypes in field-grown spring wheat. Eur. J. Plant Pathol. 127:407-417.

Vujanovic, V., Goh, Y. K., and Daida, P. 2012. Heat- and cold-shock responses in Fusarium graminearum 3 acetyl- and 15 acetyl-deoxynivalenol chemotypes. J. Microbiol. 50:97-102.

Ward, T. J., Bielawski, J. P., Kistler, H. C., Sullivan, E., and O'Donnell, K. 2002. Ancestral polymorphism and adaptive evolution in the trichothecene mycotoxin gene cluster of phytopathogenic Fusarium. Proc. Natl. Acad. Sci. USA 99:9278-9283.

Yan, W., Li, H. B., Cai, S. B., Ma, H. X., Rebetzke, G. J., and Liu, C. J. 2011. Effects of plant height on type I and type II resistance to Fusarium head blight in wheat. Plant Pathol. 60:506-512.

Yu, J., Bai, G., Cai, S., Dong, Y., and Ban, T. 2008. New Fusarium head blightresistant sources from Asian wheat germplasm. Crop Sci. 48:1090-1097.

Zhou, W. C., Kolb, F. L., Bai, G. H., Domier, L. L., and Yao, J. B. 2002. Effect of individual Sumai 3 chromosomes on resistance to scab spread within spikes and deoxynivalenol accumulation within kernels in wheat. Hereditas 137:81-89. 